\title{
O MAPA DAS HEPATITES CRÔNICAS NO BRASIL NO PERÍODO DE 2010 A 2014
}

\section{THE MAP OF CHRONIC HEPATITIS IN BRAZIL FROM 2010 TO 2014}

\author{
Cleilton Sampaio De Farias \\ Doutor em Ensino de Biociências e Saúde \\ Instituto Federal de Educação, Ciência e Tecnologia do Acre \\ cleilton.farias@ifac.edu.br \\ Ricardo Antunes Dantas de Oliveira \\ Doutor em Demografia \\ Instituto de Comunicação e Informação Científica e Tecnológica em Saúde \\ ricardo.dantas@icict.fiocruz.br \\ Maurício Roberto Motta Pinto da Luz \\ Doutor em Biologia Celular e Molecular \\ Instituto Oswaldo Cruz \\ mauluz@ioc.fiocruz.br
}

\section{RESUMO}

As hepatites virais são doenças causadas por cinco vírus distintos ( $A, B, C, D$ e $E)$, que têm em comum o forte acometimento do fígado humano. As hepatites $B, C$ e $D$ se tornam crônicas quando a inflamação do fígado se mantém pelo prazo mínimo de seis meses. Entretanto, qual é o contexto geográfico dessas doenças e qual o potencial para a cronificação de cada um desses vírus? Assim, objetivou-se mapear a distribuição dos casos notificados e taxas de prevalência das hepatites crônicas no Brasil, no período de 2010 a 2014 e apresentar algumas características do potencial para cronificação dos vírus B, C e D. Para tanto, incialmente, os dados foram coletados do Sistema de Informação de Agravos de Notificação - Sinan. Posteriormente, elaborou-se o mapa das hepatites crônicas utilizando-se o software Quantum GIS - QGIS, a projeção cartográfica ATM Datum SAD 69 e a base cartográfica do Zoneamento Ecológico Econômico do Acre e, por fim, aplicou-se técnicas de estatística exploratória de correlação linear de Pearson ( $r$ ) para analisar o potencial de cronificação. Enfim, o mapa das hepatites crônicas destacou unidades da federação nos quais essas hepatites possuem grande importância como os estados de São Paulo e Rio Grande do Sul, que apresentaram as maiores quantidades de casos confirmados enquanto o Acre apresentou a maior taxa de prevalência. Em relação ao potencial de cronificação, por mais que os resultados tenham apresentados valores acima dos mencionados na literatura, eles confirmam que os adultos, do sexo masculino ainda são os mais afetados, muito embora, as crianças possuam o maior potencial para a cronificação.

Palavras chave: Hepatites crônicas. Mapa. Brasil.

\begin{abstract}
Viral hepatitis are diseases caused by five distinct viruses (A, B, C, D and E), which have in common the strong involvement of the human liver. Hepatitis $B, C$ and $D$ become chronic when inflammation of the liver is maintained for at least six months. However, what is the geographical context of these diseases and what is the potential for the chronification of each of these viruses? Thus, the objective was to map the distribution of reported cases and prevalende rates of chronic hepatitis in Brazil, from 2010 to 2014 and to present some characteristics of the potential for the chronification of viruses B, C and D. Initially, the data were collected from the Notification Disease Information System - Sinan, and the chronic hepatitis map was then prepared using the Quantum GIS - QGIS software, the ATM Datum SAD 69 cartographic projection and the Acre Economic Ecological Zoning cartographic base. Finally, Pearson ( $r$ ) linear correlation exploratory statistical techniques were applied to analyze the potential for chronicity. Finally, the map of chronic hepatitis highlighted
\end{abstract}

Recebido em: 07/01/2020

Aceito para publicação em: 26/05/2020. 
federation units in which these diseases are extremely important, such as the states of São Paulo and Rio Grande do Sul, which have the highest number of confirmed cases and Acre in prevalence rate. Regarding the potential for chronification, even though the results have shown values above those mentioned in the literature, they confirm that male adults are still the most affected, although children have the greatest potential for chronification.

Keywords: Chronic hepatitis. Map. Brazil.

\section{INTRODUÇÃO}

\section{As hepatites virais}

As hepatites virais são doenças causadas principalmente por cinco vírus distintos, que têm em comum o acometimento do fígado humano: hepatite A causada pelo vírus A - VHA, hepatite B causada pelo vírus $B-V H B$, hepatite $C$ causada pelo vírus $C-V H C$ e hepatite $D$, ou delta, que é causada pelo vírus D - VHD e hepatite E causada pelo VHE (SILVA, 1995).

Cada forma de hepatite viral possui especificidades quanto aos meios de transmissão e contaminação. As hepatites B, C e D apresentam algumas características semelhantes como as fontes de infecção ligadas ao sangue, hemoderivados e fluídos corpóreos, bem como os modos de transmissão sexual, parenteral e percutâneo-sanguíneo. Há, porém, importantes diferenças em relação ao período de incubação, à transmissibilidade dos vírus, ao risco de estabelecimento da forma crônica e à mortalidade (FARIAS; SILVA, 2015). A transmissão sexual do VHC também tem sido relatada de forma esporádica, assim como há possibilidade de transmissão vertical, ainda que em menor proporção dos casos (FAUTEUX-DANIEL et al., 2017).

Nem sempre a infecção por esses vírus apresenta sintomas. Estes, quando presentes, incluem frequentemente febre, fraqueza, mal-estar, dor abdominal, enjoo/náuseas, perda de apetite, urina escura, icterícia (olhos e pele amarelados) e fezes esbranquiçadas (BRASIL, 2009).

\section{As hepatites crônicas}

De forma geral, "considera-se como hepatite crônica $(\mathrm{HC})$ a inflamação do fígado consequente de infecção viral que se mantém pelo prazo mínimo de seis meses" (ALVES; GAYOTTO, 1995, p. 44) e como portador crônico, o indivíduo que conserva o vírus ativo em seu organismo por tempo superior a esse período (CARRILLO e SILVA, 1995, p. 76). Segundo Alves e Gayotto (1995), do ponto de vista clínico, na maioria das vezes, é impossível caracterizar em que estágio crônico o paciente se encontra. Para tanto, é necessária a realização de biópsia hepática e a devida classificação anatomopatológica (BRASIL, 2008).

As hepatites crônicas podem ser persistentes (HCP) e ativas (HCA). As primeiras são as que comportam baixos graus de necrose em saca-bocados (NSB) e as últimas são aquelas que apresentam graus mais significativos de NSB, sendo então divididas em formas de moderada e intensa atividade (ALVES; GAYOTTO, 1995).

As hepatites $B, C$ e $D$ se tornam crônicas em proporções diferentes. Por exemplo, a hepatite $B$ apresenta de $2 \%$ a $5 \%$ de chance de se cronificar (SILVA; GRANATO,1995), já no caso da hepatite C esses valores são maiores (de $60 \%$ a $85 \%$ ) (WESTBROOK; DUSHEIKO, 2014). A hepatite D, por sua vez apresenta um amplo intervalo de $2 \%$ a $70 \%$ de possibilidade de cronificação (SILVA; GRANATO,1995).

O potencial para cronificação varia em função de alguns fatores ligados aos vírus (B, $C$ e $D)$ e outros ligados ao hospedeiro, tais como faixa etária, imunodeficiências, sexo e coinfecções.

No caso da Hepatite B, mais de $90 \%$ dos casos evoluem para cura completa. Nos $10 \%$ dos casos que não resultam em cura, define-se formalmente a hepatite crônica associada ao VHB do ponto de vista sorológico, ou seja, quando a persistência do Antígeno de Superfície da Hepatite B - AgHBs é superior a 6 meses (SILVA; GRANATO, 1995).

No entanto, a maioria dos casos crônicos em adultos evolui para a HCP e apenas $3 \%$ apresentam HCA e, por consequência, a cirrose. Porém, quando a infecção ocorre em recém-nascido o índice de cronicidade é muito elevado, podendo alcançar até 90\% (ALVES; GAYOTTO,1995). Para evitar que

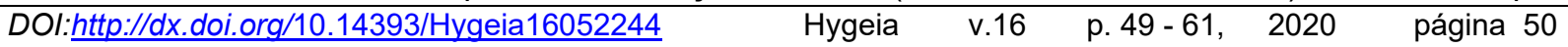


isso ocorra, há um acompanhamento específico para as gestantes que apresentam a infecção pelo VHB, tanto durante a gestação e logo após o parto, quando o recém-nascido recebe, além da vacina, a imunoglobulina contra a hepatite $\mathrm{B}$, nas primeiras 12 horas de vida para evitar a transmissão vertical (BRASIL, 2009).

Estima-se que há cerca de dois milhões de pessoas com hepatite B crônica no Brasil e destes, 220 mil estariam na região Norte. Este fato garante à hepatite $B$ o status de grave problema para a região (BRASIL, 2002; 2015; SILVA et al., 2012; WHO, 2017).

Em pesquisa recente sobre a taxa de detecção da hepatite B no período de 2008 a 2018, verificou-se que a maioria dos casos confirmados estava na forma clínica crônica, representando $72,5 \%$ do total. Os casos agudos representaram $15,8 \%$ e os fulminantes, $0,2 \%$. A porcentagem restante são dos inconclusivos e não informados (BRASIL, 2019a).

O principal vírus com potencial de cronicidade é justamente o VHC, respondendo por $70 \%$ dos casos de hepatite crônica (GHANY et al., 2009), 40\% dos casos de cirrose, $60 \%$ dos casos de carcinoma hepatocelular e $30 \%$ dos transplantes hepáticos em países industrializados (CONTE et al., 2000; VASCONCELOS et al., 2006).

No Brasil, já se chegou a estimar que entre $1 \%$ a $3 \%$ (2 a 6 milhões) da população estavam com a hepatite C crônica (CRUZ; SHIRASSU; MARTINS, 2009; STRAUSS, 2001). Atualmente a estimativa é de aproximadamente $0,7 \%$, o que corresponde a cerca de 700 mil casos que necessitam de tratamento (BENZAKEN et al., 2018). Ressalta-se que essa prevalência de $0,7 \%$ se dá em relação à população compreendida na faixa etária de 15 a 69 anos (BRASIL, 2019). De modo geral, a hepatite C é descoberta somente na fase crônica e como os sintomas são muitas vezes poucos e inespecíficos, a doença pode evoluir durante décadas sem suspeição clínica (BRASIL, 2019).

Em pesquisa recente sobre a taxa de detecção da hepatite $C$ no período de 2008 a 2018, verificou-se que a maioria dos casos confirmados forma na forma clínica crônica, representando $81,8 \%$ do total. Os casos agudos representaram $2,9 \%$ e os fulminantes, $0,2 \%$. A porcentagem restante são dos inconclusivos e não informados (BRASIL, 2019a).

A hepatite $D$, por sua particularidade de estar ligada à infecção concomitante com a hepatite $B$, é relacionada não só com a maior gravidade das hepatites virais agudas, como também com a maior progressão para a cronicidade e o desenvolvimento mais frequente e rápido da cirrose (ALVES; GAYOTTO, 1995).

Em pesquisa recente sobre a taxa de detecção da hepatite D no período de 2008 a 2018, verificou-se que a maioria dos casos confirmados estava na forma clínica crônica, representando $75,3 \%$ do total. Os casos agudos representaram $19,2 \%$ e os fulminantes, $0,5 \%$. A porcentagem restante são dos inconclusivos e não informados (BRASIL, 2019a).

Segundo Haesbaert (2004, p. 20), "não há como definir o indivíduo, o grupo, a comunidade, a sociedade sem ao mesmo tempo inseri-los num determinado contexto geográfico, territorial". Analogamente, também não há como definir as hepatites crônicas sem inseri-las em um contexto geográfico territorial, nos quais se encontram os indivíduos, os grupos, as comunidades e as sociedades que são afetadas por elas. Há que se considerar ainda o espaço das relações responsáveis pela infecção e transmissão.

Assim, o presente trabalho objetivou mapear a distribuição dos casos notificados e taxas de prevalência das hepatites crônicas no Brasil, no período de 2010 a 2014 e apresentar algumas características do potencial para cronificação dos vírus $\mathrm{B}, \mathrm{C}$ e D.

Os mapas têm sido considerados entre os principais elementos responsáveis pela renovação da Geografia da Saúde e são ainda indispensáveis na explicação espacial das doenças e de seus determinantes (BARCELLOS; BASTOS, 1996; PEITER, 2005; PINA, 1998). Além disso, as funções dos mapas na Geografia da Saúde estão cada vez mais abrangentes: eles têm sido utilizados na identificação de padrões espaço-temporais na ocorrência de doenças (BARROZO, 2014), na identificação de quem vive no local afetado pelas doenças e a quais circunstâncias estão expostas (CHEN et al., 2006) e no planejamento das ações de saúde no território (SILVEIRA; JAIME, 2014). Em síntese, os mapas servem para ligar o território com o que nele existe (WOOD, 1992), por que não dizer, então, que os mapas ligam o território com a ocorrência das hepatites crônicas? 


\section{PROCEDIMENTOS METODOLÓGICOS}

A ocorrência das hepatites crônicas foi abordada por meio da análise dos casos confirmados, que mostraram os valores absolutos e das taxas de prevalência, que apresentaram os valores relativos à população por 100.000 habitantes. Os casos confirmados foram coletados do Sistema de Informação de Agravos de Notificação - Sinan (SINAN, 2016) e transformados em taxa de prevalência de acordo com a metodologia: prevalência $=\mathrm{N}^{\circ}$ de casos de uma doença ocorridos em uma população em um determinado período / $\mathrm{N}^{\circ}$ de pessoas sob risco de desenvolver a doença durante o mesmo período $\mathrm{x}$ 100.000 (WALDMAN, 1998). Considerou-se a quantidade da população de cada unidade da federação no ano de 2012, por ser o ano que fica no meio do período pesquisado.

Segundo informações disponíveis na base do Sinan (2016), a ocorrência de hepatite crônica foi confirmada através de testes sorológicos com a presença dos marcadores específicos nos exames dos pacientes, conforme descrito no Quadro 1. No caso da hepatite C foram realizados também exames de biologia molecular complementares.

Quadro 1 - Confirmação do tipo de hepatite crônica a partir dos indicadores sorológicos

\begin{tabular}{|l|l|}
\hline Tipo de Hepatite Crônica & Marcadores serológicos \\
\hline Hepatite B & $\begin{array}{l}\text { HBsAg positivo, Anti-HBc (total) positivo e Anti-HBc } \\
\text { IgM negativo }\end{array}$ \\
\hline Hepatite C & Anti-HCV reagente e HCV-RNA detectável/positivo \\
\hline Hepatite D & $\begin{array}{l}\text { HBsAg positivo, Anti-HBc positivo, Anti-HBc IgM } \\
\text { negativo e Anti-delta reagente }\end{array}$ \\
\hline
\end{tabular}

Fonte: Sinan (2026).

O mapa de distribuição da ocorrência das hepatites crônicas foi elaborado utilizando-se o software Quantum GIS - QGIS (NANNI et al., 2014), a projeção cartográfica ATM Datum SAD 69 e a base cartográfica do Zoneamento Ecológico Econômico do Acre - ZEEAC (disponibilizada pela Unidade Central de Geoprocessamento do Acre - UCEGeo/AC).

No mapa, utilizou-se dois métodos para apresentar a ocorrência da doença: a) método das figuras coropléticas (taxas de prevalências) no qual se atribui a cada cor um valor correspondente para evidenciar a ordem visual crescente nas manifestações em área e, b) método das figuras geométricas proporcionais centralizadas (casos confirmados) que atribui a cada tamanho um valor correspondente para evidenciar a relação de proporcionalidade entre as variáveis (MARTINELLI, 2013).

Para a compreensão dos fatores ligados ao potencial para cronificação dos vírus $B, C$ e $D$, aplicou-se técnicas de estatística exploratória de correlação linear de Pearson (r). Considerou-se como variáveis dependentes os casos notificados e taxas de incidências das hepatites virais e independentes os casos confirmados e taxas de prevalência das hepatites crônicas (OLIVEIRA, 2005).

\section{O MAPA DAS HEPATITES CRÔNICAS NO BRASIL NO PERÍODO DE 2010 A 2014}

No Brasil, no período de 2010 a 2014, ocorreram 167.040 casos confirmados das hepatites virais B, C e D, com uma taxa de incidência de 86,11 por 100.000 . A distribuição dessas ocorrências pelas unidades da federação mostrou que os estados de São Paulo (46.772), Rio Grande do Sul (25.982) e Paraná (15.532) apresentaram as maiores quantidades de casos confirmados. Proporcionalmente, no entanto, as maiores taxas de incidências por (100.000) foram observadas nos estados do Acre $(607,02)$, do Rio Grande do Sul $(241,23)$ e de Santa Catarina $(199,41)$ (FARIAS; OLIVEIRA; LUZ, 2019).

Desses 167.040 casos confirmados de hepatites B, C e D, 20.820 casos $(12,5 \%)$ evoluíram para a cura na fase aguda e $146.220(87,5 \%)$ evoluíram para hepatite crônica, com uma taxa de prevalência de 75,38 (por 100.000 habitantes) (SINAN, 2016).

A distribuição dos casos confirmados e taxas de prevalências das hepatites crônicas pelas unidades da federação mostrou que os estados de São Paulo (43.674), Rio Grande do Sul (19.591) e Paraná (14.748) apresentaram as maiores quantidades de casos confirmados e proporções, enquanto os

\begin{tabular}{llllll}
\hline DOl:http://dx.doi.org/10.14393/Hygeia16052244 & Hygeia & v.16 & p. $49-61$, & 2020 & página 52
\end{tabular}


estados do Acre $(578,95)$, Santa Catarina $(184,26)$ e Rio Grande do Sul $(140,50)$ registraram taxas de prevalência (Figura 1) (SINAN, 2016).

Figura 1 - Mapa de distribuição das hepatites crônicas (casos confirmados e taxas de prevalência por 100.000 , de 2010 a 2014).

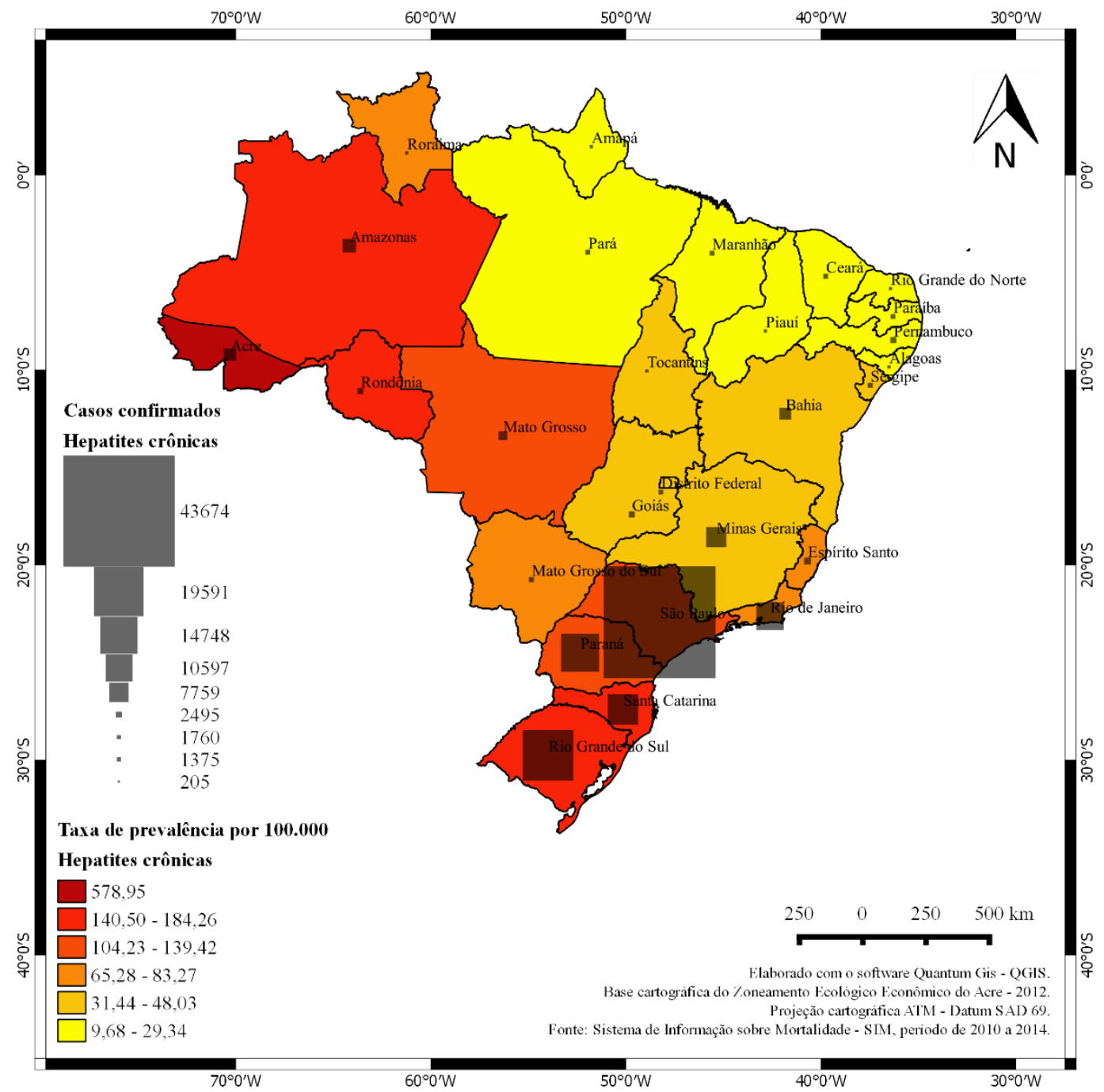

Fonte: Dados do SINAN (2016) e elaborado pelo autor.

Buscando compreender os fatores ligados ao potencial para cronificação dos vírus $B, C$ e D com as hepatites crônicas, correlacionamos os dados das hepatites crônicas com os dados das hepatites virais $B, C$ e D. Nos casos confirmados ocorreu forte correlação positiva com a etiologia $C(0,97)$ e com a B $(0,95)$, sendo nula para a etiologia $D$. Isso justifica, porque os estados de São Paulo (30.851), Rio Grande do Sul (18.866) e Paraná (5.666) apresentaram as maiores quantidades de casos confirmados de hepatites crônicas, já que foram os mesmos que apresentaram as maiores quantidades de casos confirmados de hepatite C e B (Figura 2). Entre 1999 a 2018 os estados da região Sudeste apresentaram as maiores proporções dos vírus B e C, com $34,9 \%$ e $60,0 \%$, sendo seguidos pelos estados da região Sul, com $31,6 \%$ e $26,9 \%$ respectivamente. Nesse período a taxa de infecção da hepatite C no Rio Grande do Sul foi a maior do Brasil (92 por 100 mil), seguido por São Paulo com a segunda maior taxa (38 por 100 mil) e pelo Paraná (23 por 100 mil) (BRASIL, 2019a). 
Figura 2 - Casos confirmados de hepatites virais (B, C e D) e hepatites crônicas, por unidade da federação, de 2010 a 2014.

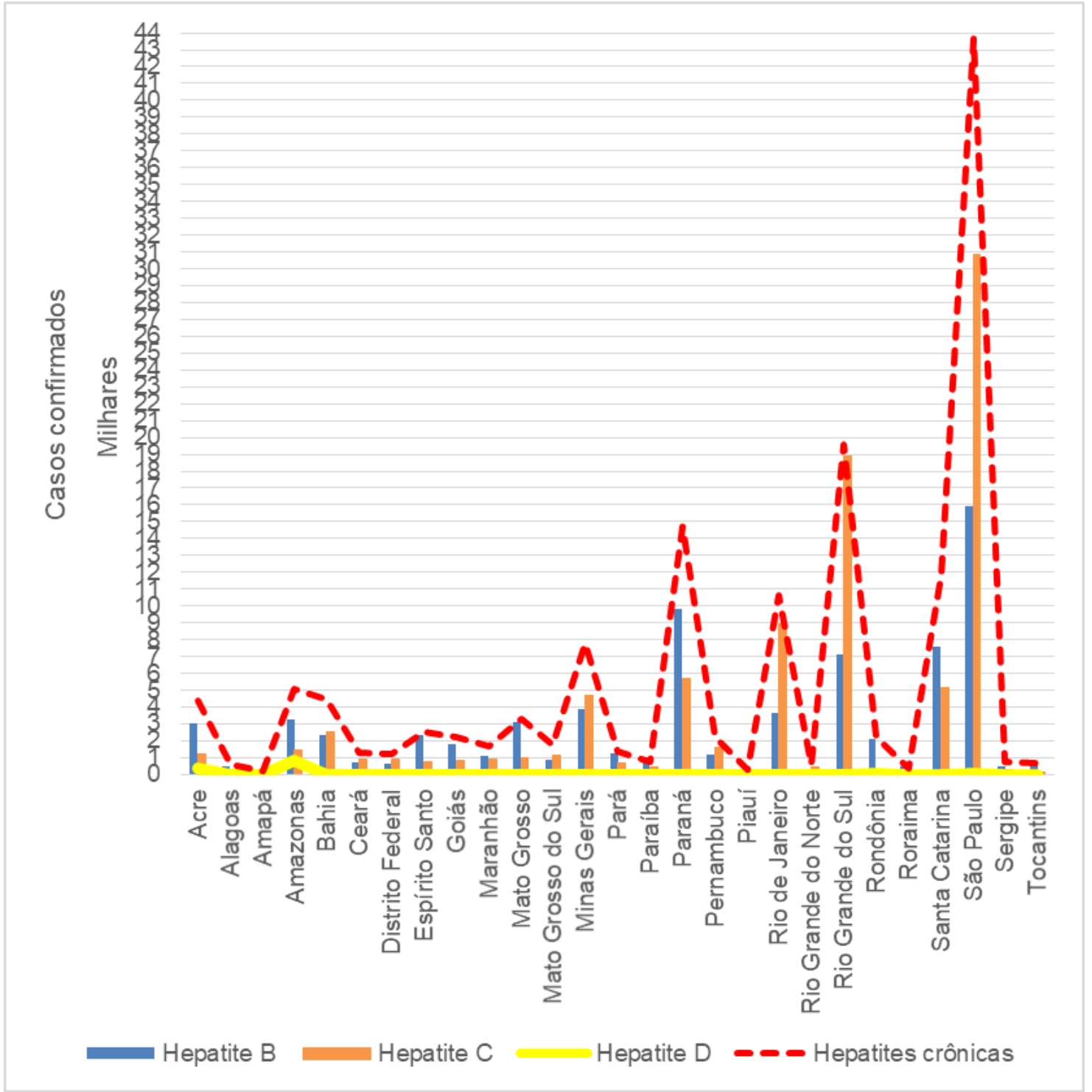

Fonte: Dados do SINAN (2016) e elaborado pelo autor.

Ao correlacionar a taxa de prevalência das hepatites crônicas com as taxas de incidência das hepatites virais, contatou-se que há forte correlação positiva com as etiologias $B(0,96)$ e $D(0,86)$ enquanto a Hepatite $C$ apresentou uma correlação positiva moderada $(0,79)$.

De fato, o estado do Acre apresentou a maior taxa de incidência de hepatite B (397,74 por 100.000) e de hepatite D (48,89 por 100.000), o estado de Santa Catarina apresentou a terceira maior taxa de incidência de hepatite B (118,29 por 100.000) e o Rio Grande do Sul apresentou a oitava maior taxa de incidência de hepatite B (66 por 100.000) (Figura 3). Esses achados estão de acordo com os descritos para o período de 2008 a 2018, segundo os quais os estados da região Norte e Sul apresentaram as maiores taxas de detecção da hepatite B acima da média nacional e os estados da região Norte as maiores proporções do vírus $D(74,9 \%)$. Nesse período a taxa de detecção da hepatite B no Acre foi a maior do Brasil (40 por 100 mil), a de Santa Catarina foi a quarta maior (18 por 100 mil) e a do Rio Grande do Sul foi a oitava (14 por 100 mil) (BRASIL, 2019a). 
Figura 3 - Taxas de - incidências das hepatites virais (B, C e D) e taxa de prevalência das hepatites crônicas, por unidade da federação, de 2010 a 2014, por 100.000.

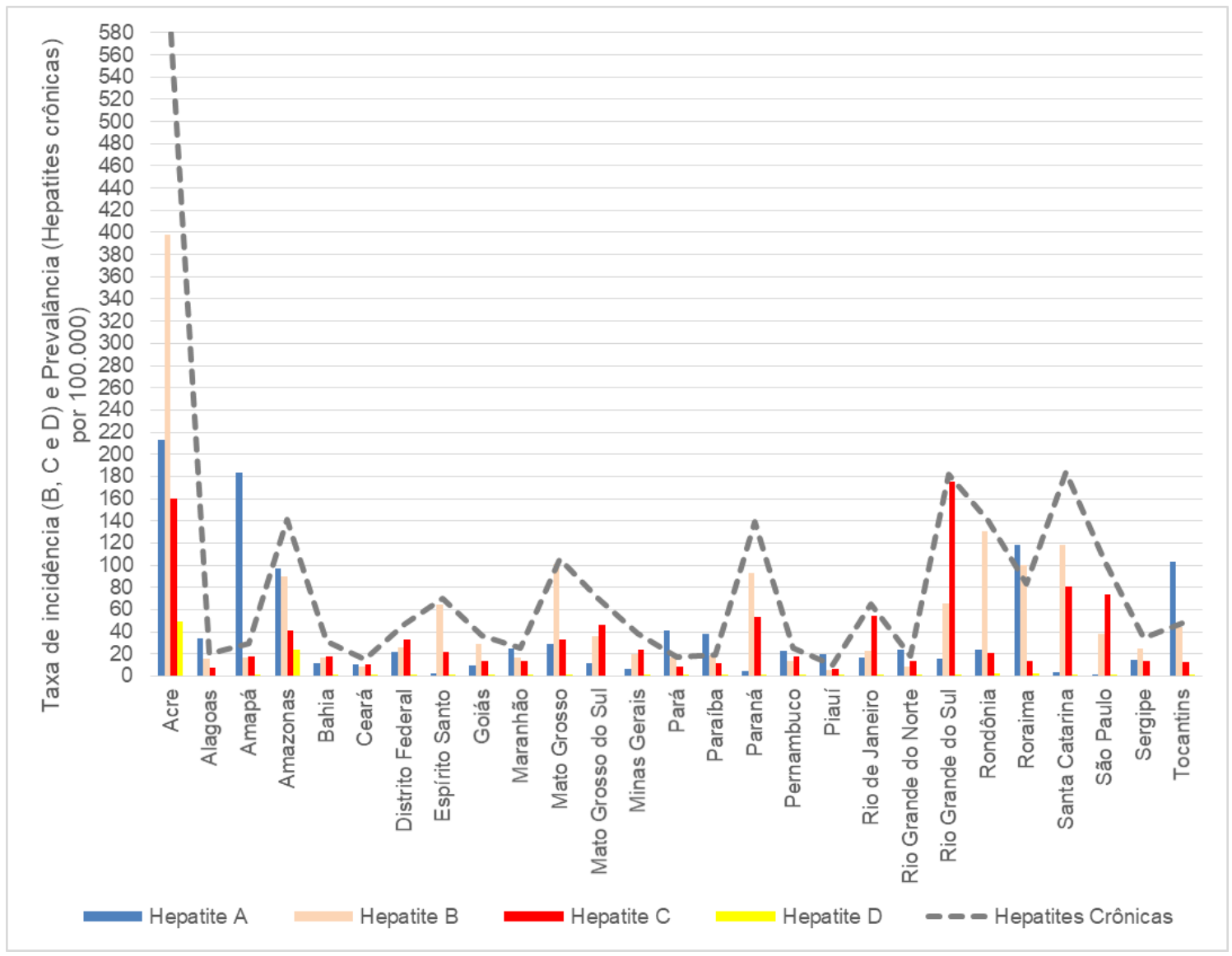

Fonte: Dados do SINAN (2016) e elaborado pelo autor.

A principais características das hepatites crônicas são mostradas na figura 4 a seguir. Destaca-se sua ocorrência predominante na população de faixa etária entre 40 a 59 anos $(47,58 \%)$ seguidas pelas pessoas de 20 a 39 anos (32,89\%)\% (Figura 4.A).

Dados recentes indicam que todas as faixas compreendidas acima de 45 anos de idade apresentaram aumento na taxa de detecção para hepatite B e que 51,2\% dos indivíduos infectados pela hepatite D possuíam idade entre 20 a 39 anos (BRASIL, 2019a).

Além disso, outra característica importante é a frequência ligeiramente maior de pessoas do sexo masculino (55,70\%) (Figura 4.B), percentual semelhante ao encontrado em uma pesquisa que analisou a taxa de detecção de casos de hepatite B, C e D segundo o sexo, entre 2008 e 2018 (Brasil 2019a). Naquele estudo ficou evidenciado que $54 \%$ dos pacientes eram do sexo masculino no caso da hepatite B, e 57,7\% no caso das hepatites C e D (BRASIL, 2019a).

Em relação a raça/cor a maioria dos casos confirmados de hepatites cônicas incidiu sobre pessoas brancas $(59,4 \%)$, seguidas pelas pardas $(30,7 \%)$, pretas $(7,9 \%)$, amarelas $(1,2 \%)$ e indígenas $(0,6 \%)$ (Figura 4.C).

O percentual obtido para a raça/cor branca é bem superior ao encontrado em outra pesquisa que foi de $46,3 \%$, seguidas das pardas $(41,2 \%)$, pretas $(10,5 \%)$, amarelas $(1,4 \%)$ e indígenas $(0,6 \%)$ (BRASIL, 2019a). Todavia, tendo-se em conta que a Hepatite C é aquela que apresenta maiores proporções de casos crônicos, essa diferença se atenua, já $58,1 \%$ dos casos crônicos daquela etiologia foram referidos como brancos, 30,9\% como pardos, 9,9\% como pretos, 0,9\% como amarelos e 0,3\% como indígenas (BRASIL, 2019a). 
Figura 4 - Distribuição percentual dos casos confirmados de hepatites crônicas B, C e D segundo sexo, faixa etária e raça cor no Brasil, 2014 a 2016.

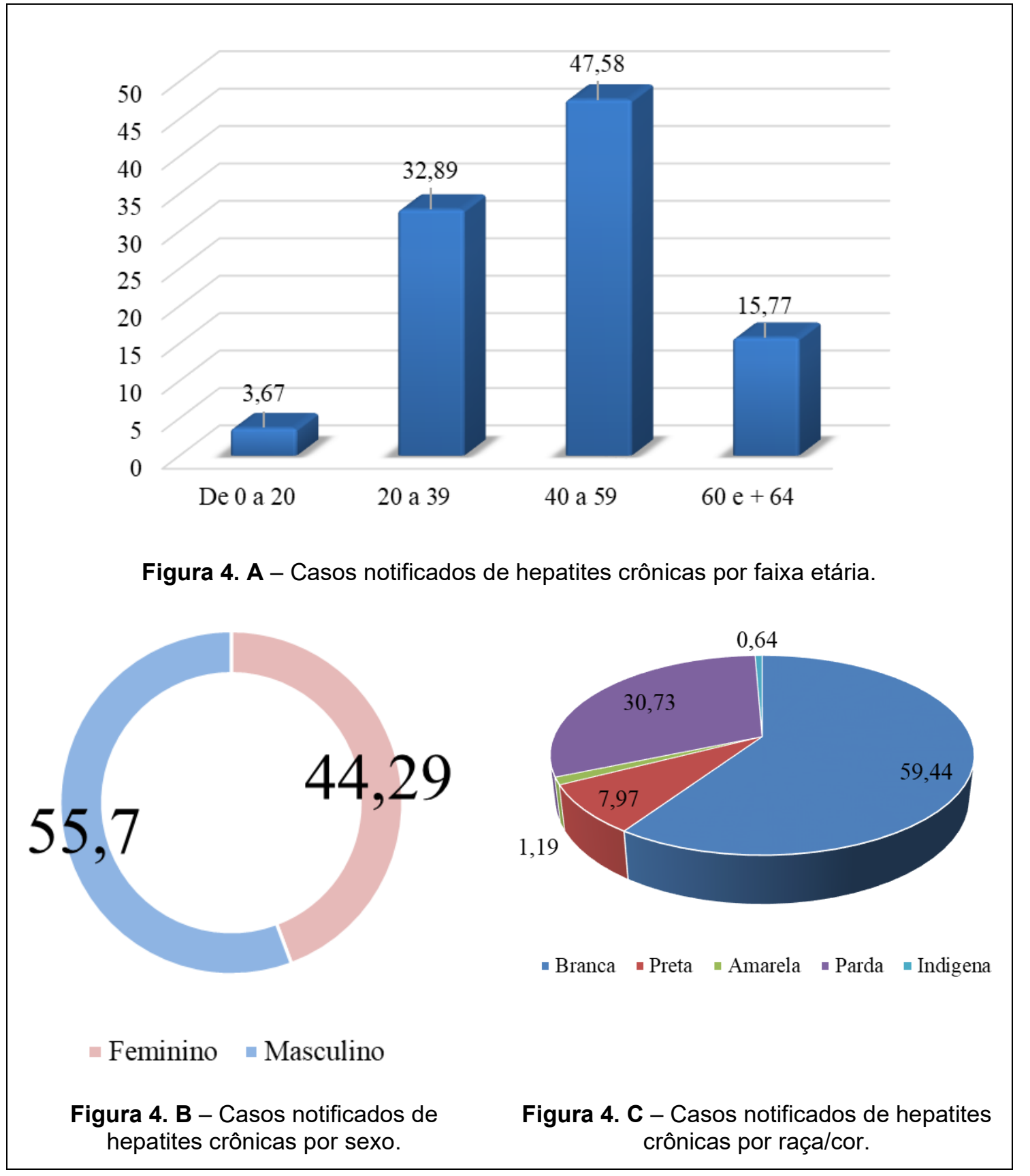

Fonte: Dados do SINAN (2016) e elaborado pelo autor.

No geral, o potencial para cronificação foi bem superior ao proposto pelo Ministério da Saúde (BRASIL, 2008) em relação as faixas etárias. Nas crianças (de 1 ano a 5 anos) foram notificados 226 casos de hepatites B, C e D dos quais 222 (98,23\%) tornaram-se crônicos. Já entre adultos jovens (de 20 anos a 39 anos) foram notificados 56.109 casos de hepatites B, C e D dois quais 48.090 se tornaram crônicos, ou seja, $85,7 \%$ cronificaram. Por fim, entre adultos mais velhos (40 a 59 anos) ocorreram 77.610 notificações das quais 69.559 (89,62\%) se tornaram crônicas.

Embora os adultos tenham uma superioridade absoluta na quantidade de notificações, foram as crianças que apresentaram o maior potencial para cronificação. 
A importância de analisar as hepatites crônicas está ligada ao fato de que os portadores crônicos serão, em princípios, os potenciais transmissores dessa enfermidade (além de indivíduos não diagnosticados e assintomáticos). Mas também serão as pessoas que sofrerão com cirrose e carcinoma hepatocelular e, por isso, demandarão atenção especial da saúde pública, sendo importante conhecer seus territórios, suas territorialidades e suas territorializações.

\section{CONCLUSÃO}

A compreensão do mapa das hepatites crônicas destacou unidades da federação nas quais essas doenças possuem grande importância e outras nas quais têm impacto menos considerável. Essa distribuição e sua heterogeneidade são resultados, sobretudo, das relações que possibilitaram historicamente o surgimento dessas enfermidades e que, em função das condições de vida e hábitos das populações, bem como da presença ou ausência e menor ou maior ação dos serviços de saúde, ainda são compartilhadas nesses espaços. Por essas questões é que alguns estados das regiões Norte, Sudeste e Sul apresentaram-se com maior importância na distribuição das hepatites crônicas e os estados das regiões Centro-Oeste e Nordeste com menor importância.

Dois estados, um da região Sudeste (São Paulo) e outro da região Sul (Rio Grande do Sul) apresentam as maiores quantidades de casos confirmados. No caso de São Paulo, por se tratar de estado populoso é natural que apresente muitos casos, já em relação ao Rio Grande do Sul isso não se aplica, por isso necessita de pesquisas complementares.

Entretanto, quando consideramos as taxas de prevalência, as hepatites crônicas discutidas no presente trabalho estão especialmente presentes no estado do Acre. Isso ocorre apesar da diversidade de agentes etiológicos e modos de transmissão que caracterizam essas hepatites. Notadamente, $\mathrm{O}$ estado além de possuir histórico dessas doenças, também apresentou altas incidências das hepatites B, C e D em suas formas não crônicas (FARIAS; OLIVEIRA; LUZ, 2019). A condição preocupante do Acre em relação às hepatites crônicas parece que tem passado despercebida na produção acadêmica nacional e internacional. Talvez isso possa ter comprometido as ações preventivas e contribuído para essas altas prevalências. Vale ressaltar a urgência de políticas públicas específicas e ações dos serviços de saúde para lidar com este agravo.

Em relação ao potencial de cronificação, os resultados, ainda que superiores aos descritos em outras fontes encontradas na literatura, eles confirmaram padrões conhecidos: predomínio de adultos do sexo masculino entre os mais afetados, ainda que as crianças possuam o maior potencial para a cronificação.

Esses resultados carecem de maiores explicações que só poderiam ser alcançadas com inquéritos realizados diretamente com algumas populações. Entretanto, é provável que as ações reduzidas de diagnóstico precoce e a falta de um tratamento eficaz possam, em conjunto, ter favorecido a permanência da infecção e sua posterior cronificação, sobretudo na região Norte, onde uma parte da população vive em áreas distantes das cidades, em que a atenção à saúde é precária. A falta de conhecimento decorrente, ao menos em parte, das altas taxas de analfabetismo e da desigualdade social presente nessas comunidades as tornam altamente propícias a propagação de doenças infecciosas e parasitárias.

\section{REFERÊNCIAS}

ALVARIZ, F. G. Hepatite C Crônica: aspectos clínicos e evolutivos. Moderna Hepatologia. 2004; Ano 30. Edição Especial: 20-32.

ALVES, V. A. F; GAYOTTO, L. C. C. Patologia das Hepatites. In: Silva, L. C. Hepatites agudas e crônicas. São Paulo: Sarvier; 1995. p. 40-55.

BARCELLOS, Christovam; BASTOS, Francisco Inácio. Geoprocessamento, ambiente e saúde: uma união possível? Cad. Saúde Públ., Rio de Janeiro, 12(3):389-397, jul-set 1996. https://doi.org/10.1590/S0102-311X1996000300012

BARCELLOS, Christovam; PEREIRA, Martha Priscila Bezerra. O território no Programa Saúde da Família. Hygeia, Uberlândia, v. 2 n. 2, p. 47-55, 2006. 
BARROZO, Ligia Vizeu. Contribuições da cartografia aos estudos de geografia da saúde: investigando associações entre padrões espaciais. Revista do Departamento de Geografia - USP, Volume Especial Cartogeo (2014), p. 413-425. https://doi.org/10.11606/rdg.v0i0.549

BASSO, Luís Alberto; RIGHI, Eléia. Casos de Hepatite A e leptospirose no município de Porto Alegre - RS entre os anos de 2007 a 2011. Hygeia 11 (20): 66 - 84, Jun/2015.

BENZAKEN, A. et al. Hepatitis C elimination by 2030 is feasible in Brazil: a mathematical modelling approach. Journal of Hepatology, [S.I.], v. 68, p. S193, 1 abr. 2018. https://doi.org/10.1016/S0168$\underline{8278(18) 30598-1}$

BRASIL. Ministério da Saúde. Programa Nacional de Hepatites Virais. Avaliação da Assistência às Hepatites Virais no Brasil. Brasília: 2002.

Ministério da Saúde. Secretaria de Vigilância em Saúde. Material instrucional para capacitação em vigilância epidemiológica das hepatites virais / Ministério da Saúde, Secretaria de Vigilância em Saúde. Brasília: Ministério da Saúde, 2008.

Ministério da Saúde. Secretaria de Vigilância em Saúde. Departamento de DST, Aids e Hepatites Virais. A B C D E das hepatites virais para agentes comunitários de saúde / Ministério da Saúde, Secretaria de Vigilância em Saúde, Departamento de DST, Aids e Hepatites Virais. Brasília: Ministério da Saúde, 2009.

Programa Nacional Para a Prevenção e o Controle das Hepatites Virais. Acesso em: 10/12/2015. Disponível no endereço: http://www.saude.gov.br/sps/areastecnicas/hepatite.htm

Protocolo Clínico e Diretrizes Terapêuticas para Hepatite C e Coinfecções. Brasília: Ministério da Saúde, 2019.

Boletim Epidemiológico - Hepatites Virais Ano VII - $\mathrm{n}^{\circ}$ 01. Brasília: Secretaria de vigilância em Saúde Ministério da Saúde, Volume 50 | № 17 | Jul. 2019a.

CARRILHO, Flair José; SILVA, Luiz Caetano da. Epidemiologia. In: SILVA, Luiz Caetano da. Hepatites agudas e crônicas. São Paulo: SARVIER, 1995.

CARVALHO, Márcia Siqueira de. ZEQUIM, Maria Angelina. Doenças infecto-contagiosas relacionada as carências habitacionais na cidade de Londrina - Paraná (Brasil). In: CARVALHO, Márcia Siqueira de. Geografia, meio ambiente e saúde em Londrina. Londrina: Edições Humanidades, 2005.

CARVALHO, Márcia Siqueira de. MENDONÇA, Francisco de Assis. Desigualdades intraurbanas e condicionantes socioambientais. Ateliê Geográfico - Goiânia-GO, v. 12, n. 1, abr/2018, p. 25-50. https://doi.org/10.5216/ag.v12i1.45132

CHEN, J.T., REHKOPF, D.H., WATERMAN, P.D., SUBRAMANIAN, S.V., COULL, B.A., COHEN, B. OSTREM, M., KRIEGER, K. Mapping and Measuring Social Disparities in Premature Mortality: The Impact of Census Tract Poverty within and across Boston Neighborhoods, 1999-2001. Journal of Urban Health: Bulletin of the New York Academy of Medicine, Vol. 83, No. 6, 1063-1084, 2006. https://doi.org/10.1007/s11524-006-9089-7

CNDSS, Comissão Nacional sobre Determinantes Sociais da Saúde. As causas sociais das iniquidades em saúde no Brasil. Ministério da Saúde, 2008.

CONTE, Vinício Paride. Carcinoma hepatocelular. Parte 1: considerações gerais e diagnóstico. Arq. Gastroenterol. São Paulo, v. 37, n. 1, p. 58-67, jan. 2000 . Disponível em $<$ http://www.scielo.br/scielo.php?script=sci_arttext\&pid=S0004-

28032000000100012\&Ing=pt\&nrm=iso>. Acesso em 27 jun. 2017. https://doi.org/10.1590/S0004$\underline{28032000000100012}$

CRUZ, C. R. B; SHIRASSU, M. M; MARTINS, W. P. Comparação do perfil epidemiológico das hepatites B e C em um serviço de São Paulo. Arq. Gastroenterol. 2009; 46(3):225-9. https://doi.org/10.1590/S0004-28032009000300016

CZERESNIA, Dina; RIBEIRO, Adriana Maria. O Conceito de Espaço em Epidemiologia: uma interpretação histórica e epistemológica. Cadernos de Saúde Pública, Rio de Janeiro, v. 16, n. 3, p. 595-613, 2000. https://doi.org/10.1590/S0102-311X2000000300002 
DANTAS, Thor Oliveira Maia. Aspectos epidemiológicos da infecção pelo vírus da hepatite $\mathrm{C}$ e coinfecções com os vírus $B$ e delta no estado do Acre, Amazônia Ocidental Brasileira. Tese (doutorado) - Universidade de Brasília, Núcleo de Medicina Tropical, 2010.

DUARTE, Paulina do Carmo Arruda Vieira; STEMPLIUK, Vladimir de Andrade; BARROSO, Lúcia Pereira (Org.). Relatório brasileiro sobre drogas. Brasília: SENAD, 2009.

EMÍLIA ARROZ, Maria. Difusão espacial da hepatite infecciosa. Finisterra. V. 14, n. 27, 1979. https://doi.org/10.18055/Finis2237

FARIA, R. M.; BORTOLOZZI, A. Espaço, território e saúde: contribuições de Milton Santos para o tema da geografia da saúde no Brasil. R. RA'E GA, Curitiba, n. 17, p. 31-41, 2009. Editora UFPR. https://doi.org/10.5380/raega.v17i0.11995

FAUTEUX-DANIEL, S. et al. Vertical Transmission of Hepatitis C Virus: Variable Transmission Bottleneck and Evidence of Midgestation In Utero Infection. Journal of Virology, [S.I.], v. 91, n. 23, dez. 2017. https://doi.org/10.1128/JVI.01372-17

FARIAS, C. S. de; SILVA, Nayara Oliveira da. Sistema de informação geográfica - SIG aplicado no estudo da geografia das hepatites virais no Acre. Revista Brasileira de Geografia Física. V. 08 N. 03 (2015) 840-847. https://doi.org/10.5935/1984-2295.20150036

; OLIVEIRA, Ricardo Antunes Dantas; LUZ, Maurício Mota Pinto da. As hepatites virais no brasil: uma análise a partir dos seus territórios. R. Ra'e Ga, Curitiba, v.46, p. $90-109$, Mar/2019. DOI: 10.5380/raega. https://doi.org/10.5380/raega.v46i1.56419

FONSECA, Carlos Ferraz da; BRASIL, Leila Melo. Infecção pelo vírus da hepatite C na região Amazônica brasileira. Revista da Sociedade Brasileira de Medicina Tropical. Hepatologia tropical. 37 (Suplemento II). 2004. https://doi.org/10.1590/S0037-86822004000700001

FONSECA, Carlos Ferraz da. Histórico das Hepatites Virais. Revista da Sociedade Brasileira de Medicina Tropical. 43(3):322-330, mai-jun, 2010. https://doi.org/10.1590/S0037$\underline{86822010000300022}$

HORNES, Karin Linete; PALHARES, José Mauro; SANTOS, Lígia Maria Rodrigues dos; PINHEIRO, Rosana. Hepatite A em Ponta Grossa (2005 - 2010): uma abordagem da Geografia da Saúde. Perspectiva Geográfica, Unioeste v.7, n.8, 2012, ISSN 1981-4801.

GHANY, M. G.; STRADER, D. B.; THOMAS, D. L.; SEEFF, L. B. Diagnosis, management, and treatment of hepatitis C: an update. Hepatology. 2009 Apr;49(4):1335-74. https://doi.org/10.1002/hep.22759

HAESBAERT, Rogério. O mito da desterritorialização: do "fim dos territórios" à multiterritorialidade. Rio de Janeiro: Bertrand Brasil, 2004.

. Território e multiterritorialidade: um debate. GEOgraphia. Ano IX - № $17-2007$.

Dilema de conceitos: espaço-território e contenção territorial. In: SAQUET, Marcos Aurélio;

SPOSITO, Eliseu Savério. (Org.). Territórios e territorialidades: teorias, processos e conflitos. São Paulo: Expressão Popular, 2009.

HORNES, Karin Linete Et Al. Hepatite A em Ponta Grossa (2005 - 2010): uma abordagem da Geografia da Saúde. Perspectiva Geográfica, Unioeste v.7, n.8, 2012, ISSN 1981-4801.

LOBATO, C. Intrafamilial prevalence of hepatites B vírus in western brasilian amazon region: Epidemiologic and biomolecular study. Journal of Gastroenterology and Hepatology. 2006. https://doi.org/10.1111/j.1440-1746.2006.04298.x

WALDMAN, E. A. Aspectos epidemiológicos da hepatite B e prognóstico de cirrose em pacientes com hepatite B crônica na Amazônia Ocidental. JSM Gastroenterol Hepatol. 3(1): 1038. 2015.

MARTINELLI, Marcelo. Mapas de geografia e cartografia temática. São Paulo: Contexto, 2013.

MONKEN, Maurício et al. O Território na Saúde: construindo referencias para análises em saúde e ambiente. In: MIRANDA, Ary (org.) Território, Ambiente e Saúde. Rio de Janeiro: Editora Fiocruz, 2008. 23-42p. 
; BARCELLOS, Christovam. Vigilância em Saúde e Território Utilizado: possibilidades teóricas e metodológicas. Cadernos de Saúde Pública, Rio de Janeiro, v. 21, n. 3, p. 898-906, 2005. https://doi.org/10.1590/S0102-311X2005000300024

NANNI, A. S; DESCOVI FILHO, L; VIRTUOSO, M. A; MONTENEGRO, D; WILLRICH, G. MACHADO, P. H; SPERB, R; DANTAS, G. S; CALAZANS, Y. Quantum GIS - Guia do Usuário, Versão 1.7.4 'Wroclaw'. Acesso em: 2014. Disponível em: http://qgisbrasil.org.br

NUNES, Heloisa Marceliano; MONTEIRO, Maria Rita de Cassia Costa; SOARES, Manoel do Carmo Pereira. Prevalência dos marcadores sorológicos dos vírus das hepatites $B$ e $D$ na área indígena Apyterewa, do grupo Parakanã, Pará, Brasil. Cad. Saúde Pública, Rio de Janeiro, 23(11):2756-2766, nov. 2007. https://doi.org/10.1590/S0102-311X2007001100023

OLIVEIRA, J. C. Biometeorologia: Efeitos dos elementos meteorológicos na qualidade e na saúde humana, estudo de casos em Maceió - AL. Fundação Manuel de Lisboa. Maceió - AL, 2005, $140 \mathrm{p}$.

PEITER, Paulo. A Geografia da Saúde na Faixa de Fronteira Continental do Brasil na Passagem do Milênio. Tese de doutorado. PPGG/UFRJ, julho de 2005.

PEREIRA, Leila Maria Moreira Beltrão; XIMENES, Ricardo Arraes de Alencar; MOREIRA, Regina Célia Moreira. (Coor.). Estudo de prevalência de base populacional das infecções pelos vírus das hepatites A, B e C nas capitais do Brasil. Pernambuco: Universidade Federal de Pernambuco, 2010.

PINA, Maria de Fátima R. P. de. Potencialidades dos sistemas de informação geográficas na área da saúde. In: NAJAR, Alberto Lopes; MARQUES, Eduardo Cesar. (org.). Saúde e espaço: estudos metodológicos e técnicas de análise. Rio de Janeiro: Editora FIOCRUZ, 1998.

PNUD, Programa das Nações Unidas para o Desenvolvimento. Atlas do desenvolvimento humano. Taxa de analfabetismo, índice de Gine e IDH-M do ano de 2010. Disponível em: http://www.atlasbrasil.org.br/2013/pt/consulta/ Acesso em: 2016.

RAFFESTIN, Claude. Por uma geografia do poder. São Paulo: Ática, 1993.

RIGOTTO, Raquel Maria; AUGUSTO, Lia Giraldo da Silva. Saúde e Ambiente no Brasil: desenvolvimento, território e iniquidade social. Cadernos de Saúde Pública, Rio de Janeiro, v. 24, supl. 4, p. 475-501, 2007. https://doi.org/10.1590/S0102-311X2007001600002

SANTOS, Milton. Metamorfoses do espaço habitado. São Paulo: Hucitec, 1988.

SAQUET, Marcos Aurélio. Por uma abordagem territorial. In: SAQUET, Marcos Aurélio; SPOSITO, Eliseu Savério. (Org.). Territórios e territorialidades: teorias, processos e conflitos. São Paulo: Expressão Popular, 2009.

SILVA, Luiz Caetano da. Conceito, tipos de hepatites por vírus e evolução dos conhecimentos. In: SILVA, Luiz Caetano da. Hepatites agudas e crônicas. São Paulo: SARVIER, 1995.

SILVA, Alessandro Lisboa da; VITORINO, Rodrigo Roger; ESPIRIDIÃO-ANTONIO, Vanderson; SANTOS, Elaine Travaglia; SANTANA, Luiz Alberto; HENRIQUE, Bruno David; GOMES, Andréia Patrícia. Hepatites virais: B, C e D: atualização. Rev Bras Clin Med. São Paulo, 2012 maijun;10(3):206-18.

SILVA; Luiz Caetano da; GRANATO, Celso F. H. Importância clínica dos marcadores virais. In: SILVA, Luiz Caetano da. Hepatites agudas e crônicas. São Paulo: SARVIER, 1995.

SILVEIRA, Heitor Matos da; JAYME, Naibi Souza. Cartografia de síntese e geografia da saúde: aproximações teóricas. Boletim geográfico de Maringá. V. 32, n. 3, p. 122-137, set.-dez., 2014. https://doi.org/10.4025/bolgeogr.v32i3.21071

SINAN, Sistema de Informação de Agravos de Notificação. Epidemiológicas e morbidade: hepatite. Informação de saúde - $\quad$ TABNET. Disponível em: $<$ http://www2.datasus.gov.br/DATASUS/index.php?area=0203\&id=29892141\&VObj=http://tabnet.data sus.gov.br/cgi/deftohtm.exe?sinannet/cnv/hepa>, Acesso em: 18/09/2016. 
SOUZA, Edevaldo Aparecido; PEDON, Nelson Rodrigo. Território e identidade. Revista Eletrônica da Associação dos Geógrafos Brasileiros - Seção Três Lagoas Três Lagoas - MS, V 1 - n. ${ }^{\circ} 6$ - ano 4, novembro de 2007.

STRAUSS, E. Hepatite C. Rev Soc Bras Med Trop. 2001;34(1):69-82. https://doi.org/10.1590/S0037$\underline{86822001000100011}$

VALLE, Suiane da Costa Negreiros do. Hepatite B e Delta: avaliação de uma série de casos na regional do Juruá - estado do Acre. Dissertação de mestrado. Universidade do estado do Amazonas - UEA. Programa de pós-graduação em medicina tropical. Manaus, 2007.

VASCONCELOS, Romes Rufino de; TENGAN, Fátima Mitiko; CAVALHEIRO, Norma de Paula; IBRAHIM, Karim; PEREIRA, Hayde Pereira; BARONE, Antonio Alci. Fatores associados às formas evolutivas graves da infecção crônica pelo vírus da hepatite C. Rev Soc Bras Med Trop. 2006;39(5):433-8. https://doi.org/10.1590/S0037-86822006000500003

VIANA, Sebastião. Estudo soroepidemiológico das Hepatites B e Delta na população de doze municípios do estado do Acre, Brasil. Tese (doutorado) - Universidade de Brasília. Faculdade de Medicina, 2003. pp. 808-814.

Alta prevalência de hepatite B e D na Amazônia Ocidental. Am. J. Trop. Med., 73(4), 2005,

WALDMAN, Eliseu Alves. Vigilância em Saúde Pública, volume 7. São Paulo: Faculdade de Saúde Pública da Universidade de São Paulo, 1998.

WESTBROOK, R. H.; DUSHEIKO, G. Natural history of hepatitis C. Journal of Hepatology, [S.I.], v. 61, n. 1, Suppl, p. S58-68, nov. 2014. https://doi.org/10.1016/i.jhep.2014.07.012

WHO, World Health Organization. Hepatitis B. World Health Organization Fact Sheet, 2016.

WHO, World Health Organization. Global hepatitis Report, 2017. ISBN 978-92-4-156545-5. Disponível em: <http://www.who.int/hepatitis/publications/global-hepatitis-report2017/en/> Acesso em: 23/10/2017 as 10 horas e 15 minutos.

WOOD, Denis. The power of maps. Routledge: London, 1992. 